\title{
Case report of an unusual hepatic abscess caused by Actinomyces odontolyticus in a patient with human immunodeficiency virus infection
}

\author{
Shao-Lun Hsu ${ }^{1,2,3^{*}}$, Chin-Ting Wu ${ }^{4}$, Yuan-Chen Chang ${ }^{3,5}$, Chia-Kwung Fan² and Yuarn-Jang Lee ${ }^{6^{*}}$ (D)
}

\begin{abstract}
Background: Actinomyces odontolyticus is not commonly recognized as a causative microbe of liver abscess. The detection and identification of $A$. odontolyticus in laboratories and its recognition as a pathogen in clinical settings can be challenging. However, in the past decades, knowledge on the clinical relevance of A. odontolyticus is gradually increasing. A. odontolyticus is the dominant oropharyngeal flora observed during infancy [Li et al. in Biomed Res Int 2018:3820215, 2018]. Herein we report a case of severe infection caused by A. odontolyticus in an immunocompromised patient with disruption of the gastrointestinal $(G l)$ mucosa.

Case presentation: We present a unique case of a patient with human immunodeficiency virus infection who was admitted due to liver abscess and was subsequently diagnosed as having coinfection of A. odontolyticus, Streptococcus constellatus, and Candida albicans during the hospital course. The empirical antibiotics metronidazole and ceftriaxone were replaced with the intravenous administration of fluconazole and ampicillin. However, the patient's condition deteriorated, and he died 3 weeks later.

Conclusion: This report is one of the first to highlight $\mathrm{Gl}$ tract perforation and its clinical relevance with $\mathrm{A}$. odontolyticus infection. A. odontolyticus infection should be diagnosed early in high-risk patients, and increased attention should be paid to commensal flora infection in immunocompromised individuals.
\end{abstract}

Keywords: Actinomyces odontolyticus, Streptococcus constellatus, Candida albicans, Liver abscess, Human immunodeficiency virus

\section{Background}

Actinomyces species are nonmotile, filamentous, anaerobic, Gram-positive, rod-shaped bacteria that are commensal flora in the dental plaque and nasopharyngeal and gastrointestinal (GI) tracts [1]. Actinomyces infection is rare and generally viewed as an opportunistic infection because it predominantly affects immunosuppressed

\footnotetext{
*Correspondence: arinahsu@gmail.com; sharonhsu1206@gmail.com ${ }^{3}$ School of Medicine, College of Medicine, Taipei Medical University, 250 Wuxing Street, Xinyi Dist., Taipei City 11031, Taiwan

${ }^{6}$ Division of Infectious Diseases, Department of Internal Medicine, Taipei Medical University Hospital, 252 Wuxing Street, Xinyi Dist., Taipei City 11031, Taiwan

Full list of author information is available at the end of the article
}

patients. Although the prognosis of Actinomyces infection is usually favorable under medical treatment, it can still lead to death in patients with extensive involvement or those without early detection. Among 25 Actinomyces species identified in the human microbiota, $A$. gerencseriae and $A$. israelii are the most commonly reported species in human diseases and are responsible for approximately $50 \%$ of cervicofacial actinomycotic infections [2, 3]. Isolation of $A$. odontolyticus from liver abscess is exceptionally rare, and early diagnosis of $A$. odontolyticus infection is considerably challenging in clinical management. Herein we present the case of a human immunodeficiency virus (HIV)-infected patient with esophageal corrosive injury who developed liver 
abscess caused by $A$. odontolyticus infection. This article is one of the first to review the limited literature on $A$. odontolyticus infection and highlight its clinical relevance with previous GI tract perforation.

\section{Case presentation}

A 45-year-old male, HIV-positive patient who was receiving regular antiretroviral therapy and had an undetectable viral load and a CD4 count of 358.31 cells $/ \mu \mathrm{L}$ presented to the emergency department with a 2-week history of fever and chills.

Two years ago, the patient developed an esophageal corrosive injury caused by drinking a strong alkali in an attempt to commit suicide. He was discharged from the hospital after receiving intensive care. Six months later, the patient developed burning and foreign body sensation in the throat. Balloon dilatation was performed due to the detection of an esophageal stricture through esophagoscopy. However, after 2 months, the patient complained of recurrent dysphagia, because of which he was dependent only on a full liquid diet. A severe esophageal stricture was detected through repeated esophagoscopy; instead of esophageal reconstruction or gastrostomy, jejunostomy was performed considering erosive injury in both the esophagus and stomach. In the following 6 months, he experienced an episode of esophagitis and lost $20 \mathrm{~kg}$ of his body weight.

Two weeks before this admission, he complained of fever and chills with dizziness, vomiting, and fatigue. On examination in the emergency room, his body weight was $40 \mathrm{~kg}$, and his BMI was 14. Vital sign assessment revealed a body temperature of $38.7^{\circ} \mathrm{C}$, a pulse rate of $139 \mathrm{bpm}$, a respiratory rate of 18 breaths per minute, a blood pressure of $95 / 67 \mathrm{mmHg}$, and oxygen saturation of $98 \%$.
Tenderness over the right upper quadrant of the abdomen was noted.

Leukocytosis with a WBC count of $12.81 \times 10^{3} / \mu \mathrm{L}$ (reference range: $4 \times 10^{3} / \mu \mathrm{L}$ to $11 \times 10^{3} / \mu \mathrm{L}$ ) with a left shift and an elevated C-reactive protein level of $22.68 \mathrm{mg} / \mathrm{dL}$ (reference range $<0.5 \mathrm{mg} / \mathrm{dL}$ ) were observed. The hemoglobin level was $6.9 \mathrm{~g} / \mathrm{dL}$ (reference range 13.0-17.0 g/ $\mathrm{dL}$ ). The platelet count was $634 \times 10^{3} / \mu \mathrm{L}$ (reference range $130-400 \times 10^{3} / \mu \mathrm{L}$ ) with a prolonged prothrombin time. The prothrombin time/international normalized ratio was 1.55 (reference range 0.78-1.12), and activated partial thromboplastin time was $46.0 \mathrm{~s}$ (reference range 32.0-45.1 s). Liver function test levels were elevated, with the glutamic oxaloacetic transaminase level being up to $129 \mathrm{U} / \mathrm{L}$ (reference range $<40 \mathrm{U} / \mathrm{L}$ ). Hypoalbuminemia was noted, with the albumin level being $2.2 \mathrm{~g} / \mathrm{dL}$ (reference range: $3.5-5.2 \mathrm{~g} / \mathrm{dL}$ ). The lactate level was $19.9 \mathrm{mg} /$ $\mathrm{dL}$ (reference range: $4.5-19.8 \mathrm{mg} / \mathrm{dL}$ ). The CD4+ count was 358.31 cells $/ \mu \mathrm{L}$ (reference range: 404.00-1612.00 cells $/ \mu \mathrm{L})$.

Chest X-ray revealed an air-fluid level beneath the right diaphragm (Fig. 1A). Abdominal computed tomography (Fig. 1B) demonstrated a multiloculated cystic lesion occupying the entire left lobe of the liver. Contrast collection within the gastric tube and esophagus revealed leakage of contrast medium from the distal esophagus suture that was possibly connected with liver abscess. A total of $160 \mathrm{~mL}$ of the purulent exudate was drained from liver cysts. Under the impression of liver abscess with impending septic shock, an intravenous fluid challenge with an inotropic agent and the empirical antibiotics metronidazole and ceftriaxone were administered immediately.

Candida albicans, Streptococcus constellatus, and A. odontolyticus were isolated (Fig. 1C), and their presence
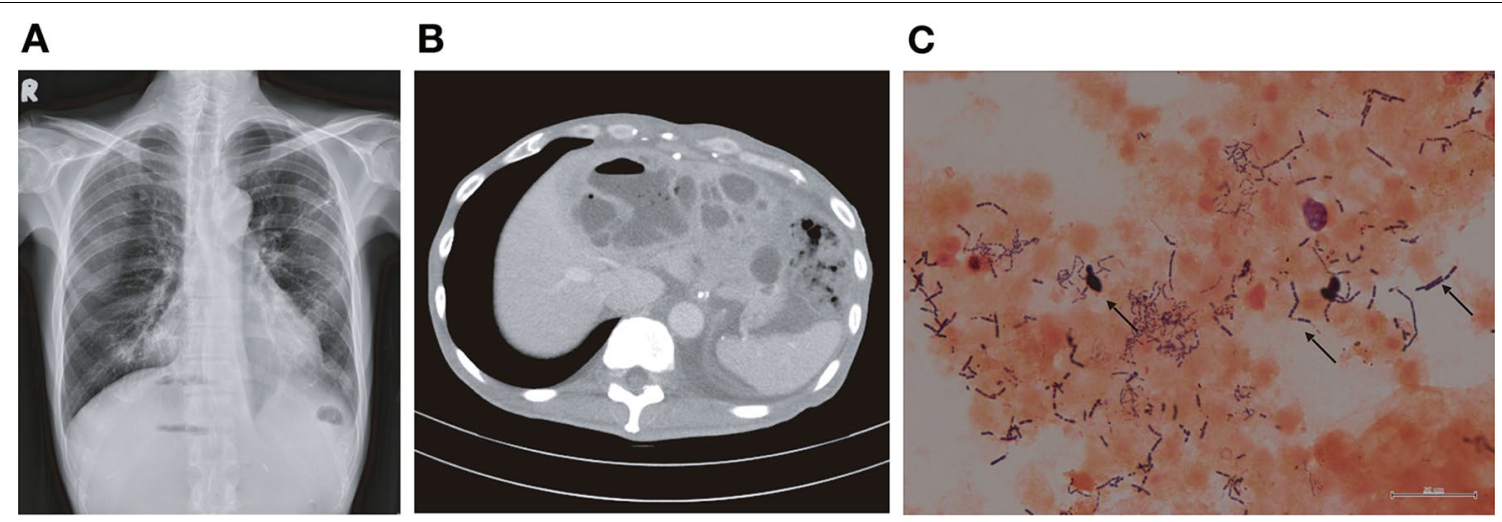

Fig. 1 Clinical image and laboratory findings suggest a polymicrobial infection in liver abscess from an HIV-infected patient. A Chest X-ray obtained on the patient's first hospitalization day revealed an air-fluid level beneath the right diaphragm, and $\mathbf{B}$ contrast-enhanced computed tomography of the abdomen demonstrated a $14 \times 7.6-\mathrm{cm}$ multiloculated cystic lesion at the level of S2, S3, and S4 in the liver parenchyma. The lesion presented with an air-fluid level and multiple enhancing septa. C Gram staining of the specimen aspirated from liver abscess revealed the budding yeast, long-chain Gram-positive coccus, and Gram-positive bacilli without branching (from left to right by the arrows) 
was confirmed through matrix assisted laser desorption ionization-time of flight (MALDI-TOF) mass spectrometry 7 days later. The presence of $A$. odontolyticus was reconfirmed based on the negative results of oxidase and catalase tests. Metronidazole and ceftriaxone were replaced with the intravenous administration of fluconazole and ampicillin. However, the patient's condition deteriorated, and he died 3 weeks later.

\section{Discussion and conclusion}

Actinomyces species are facultatively anaerobic, Grampositive, rod-shaped bacteria that are the commensal flora of the oral cavity, nasopharyngeal tract, GI tract, and skin [1]. They mostly affect immunodeficient or immunocompromised individuals who have other underlying diseases [1]. Once the anatomical barrier is disrupted, Actinomyces accompanied with other pathogenic bacteria in the alimentary tract may invade into adjacent tissues and cause infection [1,3]. Actinomycosis lesions in humans usually consist of other aerobic or anaerobic species such as A. actinomycetemcomitans, Eikenella corrodens, Capnocytophaga, Fusobacteria, Bacteroides, Staphylococci, Streptococci, and Enterobacteriaceae [4].

In the present case, corrosive injury throughout the alimentary tract and jejunostomy were considered potential predisposing factors contributing to infection caused by oropharyngeal flora including C. albicans, S. constellatus, and $A$. odontolyticus.

Valour et al. [3] reported that $A$. gerencseriae and $A$. israelii are the dominant species isolated from $70 \%$ of human forms of actinomycosis. Isolation of $A$. odontolyticus from liver abscess is exceptionally rare. Moreover, Actinomyces species such as A. odontolyticus, A. meyeri, and $A$. graevenitzii have been reported to be isolated from the oral cavity (including the tongue surface, tonsillar crypts, and distal esophagus [1]) in one-third of infants at the age of 2 months, and the diversity of Actinomyces increases with age [5]. In particular, A. odontolyticus is the predominant species in the oral cavity of edentulous infants and is the only representative of the genus found at an early age $[5,6]$.

In our patient, the repair and regeneration of the oral mucosa were similar to those observed in edentulous infants, which may be the reason for the unusual infection caused by A. odontolyticus instead of other Actinomyces species.

Actinomyces odontolyticus resides on mucosal surfaces and gains access into the deeper tissue through trauma [1]. Previous esophageal corrosive injury as well as hypoimmunity may have contributed to the severe infection in our patient. Few reports of A. odontolyticus infection shared similar characteristics with our case.
In their case report, Považan et al. described $A$. odontolyticus-associated bacteremia in a patient on the 10th hospital day after chemotherapy treatment [7]. A study by Cone et al. summarized 25 cases of $A$. odontolyticus infection from 1974 to 2003, and five of the infected patients were immunosuppressed [8]. Lopes et al. described peritonitis caused by $A$. odontolyticus in a patient with underlying gastric perforation [9]. Furthermore, Deivert et al. reported hepatic actinomycosis in an immunocompetent patient who had undergone endoscopic and surgical treatment due to hepatic artery pseudoaneurysm. They presumed that previous interventions provided a portal of entry for bacteria, leading to indolent abscess formation [10].

The application of prolonged therapies with high doses of amoxicillin or penicillin $G$ remains the firstline treatment against Actinomyces. However, some studies have suggested tetracyclines or doxycycline as alternative regimens $[11,12]$. Abscess drainage was performed in patients with widespread necrotic tissues or those nonresponsive to antibiotic therapy. In some cases, blood supply to infected sites was insufficient for antibiotics to penetrate; consequently, surgery was considered [11]. However, aggressive treatment was not favored in our patient considering his asthenia condition and personal reasons.

In summary, actinomycosis is still a disease that poses a considerable diagnostic challenge in clinical settings. Coinfection of oropharyngeal flora including C. albicans, S. constellatus, and A. odontolyticus is rarely reported. In the present case, a history of GI tract perforation provided crucial clues toward unidentified bacterial diseases among immunocompromised individuals. With the increasing availability of molecular identification techniques, such as MALDITOF, the reported number of cases of $A$. odontolyticus infection is likely to increase. In conclusion, $A$. odontolyticus should be considered as a causative pathogen in patients with disruption of the GI mucosa. We emphasize the importance of early diagnosis, and delays in treatment can lead to increased morbidity and mortality.

\section{Acknowledgements \\ We thank the study participants, Chin-Ting Wu for assistance with figures, and staff in the medical laboratory department of Taipei Medical University (TMU) who assisted in performing the confirmation test. In addition, we thank Yuarn- Jang Lee and the clinical workers of TMU infectious diseases for their contribu- tion to the clinical care of the patient and Yuan-Chen Chang for her assistance in obtaining the approval of the TMU-Joint Institutional Review Board (Grant No: N201911019). This manuscript was edited by Wallace Academic Editing, we thank their efforts in revised and improved the manuscript.}

\section{Authors' contributions}

Authors contributed to the manuscript in the following manner: Conception and design: YJL, SLH. Collection and assembly of data: YJL, SLH, and CTW. Data analysis and interpretation: YJL, SLH, and CTW. Manuscript writing: SLH, 
CTW, and YJL. Final proofreading and revision of the manuscript: YJL, YCC, and CKF. All authors read and approved the final manuscript.

\section{Funding}

This work did not receive financial support or any other relevant funding

\section{Availability of data and materials}

The datasets used during the current study are available from the corresponding author on reasonable request.

\section{Declarations}

\section{Ethics approval and consent to participate}

This study was approved by the TMU-Joint Institutional Review Board, Taipei, Taiwan. (TMU-JIRB No.: N201911019).

\section{Consent for publication}

Written informed consent was obtained from the patient's next of kin for publication of this case report and any accompanying images. Related information is available for review by the Editor-in-Chief of this journal.

\section{Competing interests}

Each of the authors has no conflicts of interest or financial ties to disclose.

\section{Author details}

${ }^{1}$ Medical Education Department, Taipei Medical University Hospital, Taipei, Taiwan. ${ }^{2}$ Department of Molecular Parasitology and Tropical Diseases, School of Medicine, College of Medicine, Taipei Medical University, Taipei, Taiwan. ${ }^{3}$ School of Medicine, College of Medicine, Taipei Medical University, 250 Wuxing Street, Xinyi Dist., Taipei City 11031, Taiwan. ${ }^{4}$ Center for Infection Control, National Taiwan University Hospital, Taipei, Taiwan. ${ }^{5}$ Medical Education Department, Chang Gung Memorial Hospital, Linkou Medical Center, Taoyuan, Taiwan. ${ }^{6}$ Division of Infectious Diseases, Department of Internal Medicine, Taipei Medical University Hospital, 252 Wuxing Street, Xinyi Dist., Taipei City 11031, Taiwan

Received: 11 December 2020 Accepted: 16 September 2021

Published online: 23 September 2021

\section{References}

1. Li J, Li Y, Zhou Y, Wang C, Wu B, Wan J. Actinomyces and alimentary tract diseases: a review of its biological functions and pathology. Biomed Res Int. 2018;2018:3820215.

2. Könönen $\mathrm{E}$, Wade WG. Actinomyces and related organisms in human infections. Clin Microbiol Rev. 2015;28(2):419-42.

3. Valour F, Sénéchal A, Dupieux C, Karsenty J, Lustig S, Breton P, et al. Actinomycosis: etiology, clinical features, diagnosis, treatment, and management. Infect Drug Resist. 2014;7:183-97.

4. Schaal KP, Lee HJ. Actinomycete infections in humans-a review. Gene. 1992;115(1-2):201-11.

5. Sarkonen N, Könönen E, Summanen P, Kanervo A, Takala A, JousimiesSomer H. Oral colonization with Actinomyces species in infants by 2 years of age. J Dent Res. 2000;79(3):864-7.

6. Dewhirst FE, Chen T, Izard J, Paster BJ, Tanner ACR, Yu WH, et al. The human oral microbiome. J Bacteriol. 2010;192(19):5002-17.

7. Považan A, Vukelic A, Secen N, Sazdanic-Velikic D, Bursac D. Actinomyces odontolyticus — associated bacteremia. Macedonian J Med Sci. 2012;5(3):324-7.

8. Cone LA, Leung MM, Hirschberg J. Actinomyces odontolyticus bacteremia. Emerg Infect Dis. 2003;9(12):1629-32.

9. Lopes VN, Dantas MJ, Andrade P, Pinto-de-Sousa J. Secondary peritonitis by Actinomyces odontolyticus. Porto Biomed J. 2017;2(5):174-5.

10. Deivert DE, Potteiger CE, Komar M, Dubagunta S, Meschter S. Primary hepatic Actinomycosis: case report and literature review. Pract Gastroenterol. 2007;31(4):92-100.

11. Adar R, Antebi E, David R, Mozes M. Abdominal actinomycosis. Israel J Med Sci. 1972;8(2):148-53.

12. Steininger $C$, Willinger $B$. Resistance patterns in clinical isolates of pathogenic Actinomyces species. J Antimicrob Chemother. 2016;71(2):422-7.

\section{Publisher's Note}

Springer Nature remains neutral with regard to jurisdictional claims in published maps and institutional affiliations.
Ready to submit your research? Choose BMC and benefit from:

- fast, convenient online submission

- thorough peer review by experienced researchers in your field

- rapid publication on acceptance

- support for research data, including large and complex data types

- gold Open Access which fosters wider collaboration and increased citations

- maximum visibility for your research: over $100 \mathrm{M}$ website views per year

At BMC, research is always in progress.

Learn more biomedcentral.com/submissions 